\title{
Giant Pulses in Millisecond Pulsars
}

\author{
B. C. Joshi
}

National Center for Radio Astrophysics, Ganeshkhind, Pune, India

M. Kramer, A. G. Lyne, M. A. McLaughlin

Jodrell Bank Observatory, University of Manchester, Macclesfield, UK

I. H. Stairs

Department of Physics and Astronomy, University of British Columbia, 6224 Agricultural Road, Vancouver, B.C. V6T 1Z1, Canada

\begin{abstract}
Giant pulses (GPs), occasional individual pulses with an intensity 100 times the average intensity, have been detected in four pulsars to date. Their origin is not well understood, but studies suggest a connection between the strength of magnetic field at the light cylinder $B_{l c}$ and the existence of GPs. Here, we report on detection of significant Large Amplitude Pulses (LAPs) in two more pulsars with high values of $B_{l c}$, PSRs J0218+4232 and B1957+20, observed using Giant Meterwave Radio Telescope (GMRT).
\end{abstract}

\section{Introduction}

Giant pulses (GPs) have been reported in four pulsars (B0531+21, B1937+21, B1821-24 and B0540-69) to date (Staelin \& Reifenstein 1968; Lundgren et al. 1995; Cognard et al. 1996; Romani \& Johnston 2001; Johnston \& Romani 2003). Three of these pulsars are millisecond pulsars (MSPs) and also show strongly pulsed hard X-ray profiles (Takahashi et al. 2001). The radio GPs occur in a narrow phase window close to the high energy non-thermal pulse indicating a common magnetospheric origin. All these pulsars have a high value of $B_{l c}$. We have used the GMRT to search for GPs in MSPs with non-thermal high energy emission and a range of $B_{l c}$ and report detection of such pulses in two more pulsars, PSRs J0218+4232 and B1957+20.

We obtained about $3600 \mathrm{~s}$ of data on each pulsar using 20 to 22 GMRT antennae in an incoherent mode at $610 \mathrm{MHz}$ with $16 \mathrm{MHz}$ of bandwidth. The expected rms noise in the above configuration of GMRT for a sampling time of $258 \mu s$ is about $1 \mathrm{Jy}$. The data in two subbands ( $8 \mathrm{MHz}$ each) were dedispersed to a common sky frequency $(610 \mathrm{MHz})$. The periods with a peak greater than $3.5 \times \mathrm{rms}$ in both bands at the same sample were identified as Large Amplitude Pulses (LAPs). This procedure disperses any narrow interference spike, discriminating against interference. A number of marginal LAPs, i.e., pulses with a peak between 3.0 to 3.5 times $\mathrm{rms}$, were also identified. 

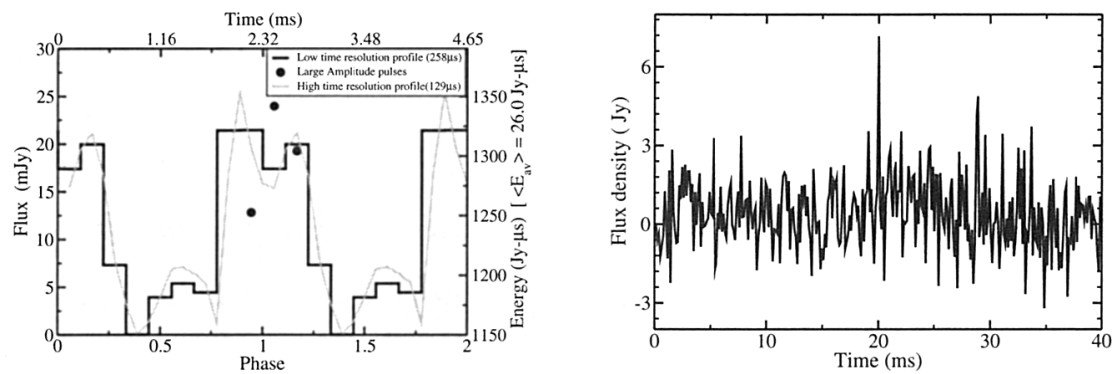

Figure 1. LAPs in PSR J0218+4232 and B1957+20. Left panel: baseline-subtracted radio integrated profile for PSR J0218+4232 with the LAPs marked with the filled circles at the phase of their occurrence. The energy in the LAP is labeled on the right. Right panel: the GP detected in PSR B1957+20 after removing the off-pulse mean.

\section{Results}

We searched 2.2 million periods for PSR J0218+4232 $\left(B_{l c} \sim 3.2 \times 10^{5} \mathrm{G}\right)$ and found three significant LAPs. Figure 1 (left panel) shows the integrated profile for this pulsar and the detected LAPs, marked with filled circles. The largest of these had an intensity 51 times the mean intensity (intensity of LAP, $I_{l a p} \sim$ $1341 \mathrm{Jy} \mu \mathrm{s}$ ). Our data also contained nine marginal LAPs, seven of which occur between phase $0.89-1.2$, which is the phase interval corresponding to one of the high energy peaks. We searched about 1 million periods for PSR B1957+20 $\left(B_{l c} \sim 3.8 \times 10^{5} \mathrm{G}\right)$ and found one significant LAP, shown in Figure 1 (right), with an intensity 129 times the mean intensity ( $\left.I_{l a p} \sim 925 \mathrm{Jy} \mu \mathrm{s}\right)$. In addition, we also detected five marginal LAPs.

PSRs B1957+20 and J0218+4232 have the fourth and sixth highest values of $B_{l c}$, respectively, among known radio pulsars. Hence, these new detections support a connection between the magnitude of $B_{l c}$ and the existence of GPs and LAPs. The data for pulsars with $B_{l c} \lesssim 10^{5} \mathrm{G}$ are still being analyzed.

\section{References}

Cognard, I., Shrauner, J. A., Taylor, J. H., \& Thorsett, S. E. 1996, ApJ, 457, 81

Johnston, S., \& Romani, R. W. 2003, private communication

Lundgren, S. C., Cordes, J. M., Ulmer, M., Matz, S. M., Lomatch, S., Foster, R. S., \& Hankins, T. 1995, ApJ, 453, 433

Romani, R. W., \& Johnston, S. 2001, ApJ, 557, L93

Staelin, D. H., \& Reifenstein, E. C. 1968, Science, 162, 1481

Takahashi, M., et al. 2001, ApJ, 554, 316 